\title{
Surface plasmon resonances of clustered
}

\section{nanoparticles}

\author{
Titus Sandu, ${ }^{*, \dagger, \S}$ Daniel Vrinceanu, ${ }^{*}$ and Eugen Gheorghiu ${ }^{\mathbb{I l}}$ \\ National Institute for Research and Development in Microtechnologies, Bucharest, Romania, \\ Department of Physics, Texas Southern University, Houston, Texas 77004, USA, and \\ International Center for Biodynamics, Bucharest, Romania \\ E-mail: titus.sandu@imt.ro
}

\begin{abstract}
Linear clusters made by tightly connecting two or more metallic nanoparticles have new types of surface plasmon resonances as compared to isolated nanoparticles. These new resonances are related to the size of the junction and to the number of interconnected particles and have direct interpretation as eigenmodes of a Boundary Integral Equation (BIE). This formulation allows effective separation of geometric and shape contribution from electric properties of the constituents. Results for particles covered by a thin shell are also provided. In addition, the present analysis sheds a new light on the interpretation of recent experiments from literature.
\end{abstract}

The interaction of electromagnetic fields with discrete systems ranging from plasmonic nanoparticles $\frac{1,2}{\underline{2}}$ to biological cells $\underline{\underline{3}}$ is governed by their polarizabilities and is strongly dependent on both dielectric and geometric properties of particles and surrounding medium. Metallic nanoparticles

\footnotetext{
${ }^{*}$ To whom correspondence should be addressed

†National Institute for Research and Development in Microtechnologies, Bucharest, Romania

Department of Physics, Texas Southern University, Houston, Texas 77004, USA

II International Center for Biodynamics, Bucharest, Romania

$\S$ International Center for Biodynamics, Bucharest, Romania
} 
such as noble-metal nanostructures exhibit the localized surface plasmon resonance (LSPR) phenomenon when the electromagnetic radiation interacts with the collective oscillations of the conduction electrons. $\stackrel{1}{-}$ The resonances in visible (VIS) and near-infrarred (NIR) induce extremely strong fields confined to the proximity of the surface of metallic nanoparticles, and therefore creating the opportunity for highly sensitive sensors, $\stackrel{4}{4}$ surface enhanced Raman scattering (SERS), $\underline{5}$ surface enhanced infrared scattering (SEIRS), $\underline{6}$ near-field microscopy, ${ }^{7}$ photoluminescence, $\stackrel{8}{,}$ second or higher harmonic generation, $\stackrel{9,10}{ }$ and other applications. Closely spaced or nearly touching (connected) nanoparticles $\stackrel{11-13}{-13}$ as well as clustered particles in different configurations $\underline{14}$ provide additional enhancements of the field within the junction region. Moreover, the "dumbbell" shaped particles have also shown other remarkable properties like the large red-shift in the dipolar response, $\stackrel{12,15}{12}$ and large variations in the spectral response. ,, $12^{.12}$

We demonstrate the spectral BIE approach by calculating the dielectric response for clusters made of up to four rotationally symmetric particles of two kinds: elongated particles, longer along the rotation axis (aspect ratio greater than unity) and flatten particles, shorter along the rotation axis (aspect ratio less than unity). [figure][1][]1] shows the imaginary part of the polarizability for such clusters, when the electric field is along the rotation axis. Resonances in the mid-infrared (mid-IR) region of the spectrum appear for both kinds of clusters and are associated with the joints between the particles. These resonances are enhanced as the relative size of the joints become smaller and they. $\frac{3}{}$ The additional resonance for clusters of more than one flatten particles (sub-figure b) appears because the cluster as a whole becomes elongated. This resonance is visible for clusters made of any number of elongated particles (sub-figure a). There are no new resonances introduced when the number of particles increases. In recent works $9,13,15$ the distinct optical behavior of touching dimers has been attributed to the joints as soon as the particles come in conductive contact in the dimer. $\stackrel{13}{ }$ Our results indicate that the resonances due to the joints are located far in mid-IR region of the optical spectrum. On the other hand, in the papers cited above,,$\frac{9,13,15}{}$ the shape of particles used in numerical simulations was close to spherical shape, which is the border shape of flatten particles. This observation together with the results of [figure][1][]1 suggests that the red- 
shifted plasmon resonance observed in $9,13,15$ for dimers is the additional resonance which appears in clusters made of flatten particles.

The idea of treating surface plasmons as electrostatic resonances has been introduced in $\underline{16}$ by showing that source-free electromagnetic fields correspond to the eigenvalues of the BIE. $\frac{17}{}$ Using a similar approach, we show that a spectral representation of BIE provide the dielectric responses of homogeneous nanoparticles and nanoshells in compact forms, which can be directly used in designing plasmonic structures. The design of analyte sensing is given as an example, where the explicit equations of plasmon resonances are used to calculate the refractive index sensitivity for each eigenmode.
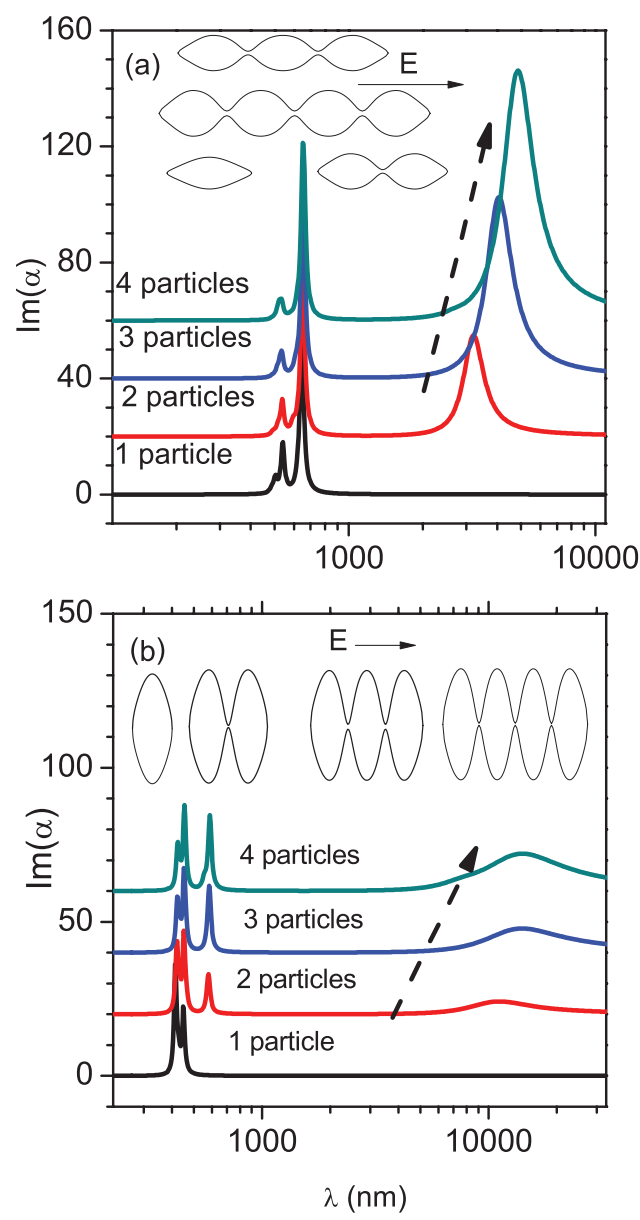

Figure 1: Imaginary part of polarizabily for clusters made of elongated (a) and flatten (b) particles in longitudinal fields. Mid-IR resonances are associated with the interparticle joints and become more prominent as the joints tighten. Resonances in the visible spectrum are associated with the shape and with the number of particles. 
Each eigenmode of the linear response operator has a contribution in the expression of polarizability proportional with its coupling weight and inversely proportional with the depolarization factor. Although this property has been demonstrated for biological cells, $\frac{3}{3}$ it is also valid for metallic nano-particles and nanoshells. If an eigenvalue has a non-zero weight and is close to the value $1 / 2$, then it is responsible for a mid-infrared (mid-IR) plasmon resonances, which can be used in optical nanoantennas. ${ }^{20,21}$ As the interparticle junction tightens, the eigenvalue approaches $1 / 2$ and the resonance is enhanced. The coupled-particle LSPR occurs at a frequency red-shifted from that of a single-particles. $\frac{2}{2}$

The nanoparticle clusters are considered under the electrostatic (quasistatic) approximation, 22 when their size is no more than one tenth of the wavelength of the incoming radiation. The optical behavior of metallic nanoparticles $\frac{16,17}{}$ as well as the dielectric behavior of living cells ${ }^{\frac{3}{}}$ can therefore be obtained from a solution of the Laplace equation derived from the corresponding BIE written for the surface $\Sigma$ which defines the particle. The polarizability of such an object of permittivity $\varepsilon_{i}$ embedded into a medium of permittivity $\varepsilon_{o}$ is given in, $\underline{3.17}$ and has the following spectral representation

$$
\alpha=\sum_{k} \frac{p_{k}}{1 /(2 \lambda)-\chi_{k}}
$$

Here electric parameter $\lambda=\left(\varepsilon_{i}-\varepsilon_{o}\right) /\left(\varepsilon_{i}+\varepsilon_{o}\right)$, while $\chi_{k}$ and $p_{k}$ are eigenvalues and coupling weights corresponding to the $k$-th eigenmode of the linear response operator $M$. This geometric operator depends only on the shape of the particle, is defined by

$$
M[\mu]=\frac{1}{4 \pi} \oint_{\Sigma} \frac{\mathbf{n}(\mathbf{x})(\mathbf{x}-\mathbf{y})}{|\mathbf{x}-\mathbf{y}|^{3}} d \Sigma(\mathbf{y})
$$

and have the following properties: ${ }^{3,16}$ its spectrum is discrete, real and bounded to the interval [$1 / 2,1 / 2]$; the value $1 / 2$ is always an eigenvalue, and it has only one active eigenvalue for spherical particles. The imaginary part of the polarizability $\alpha$ is directly related to optical absorption of metallic nanoparticles, $\stackrel{22}{2}$ and depends on the polarization of the incident radiation ${ }^{3,17}$ through dipole-coupling weights $p_{k}$. Equation 1 shows how material properties, all collected within $\lambda$, are 
separated from geometric properties contained in $\chi_{k}$ and $p_{k}$.

An object covered by a very thin shell of permittivity $\varepsilon_{S}$ has a polarizability calculated from ?? by replacing $\lambda$ by $\lambda_{k}=\left(\tilde{\varepsilon}_{k}-\varepsilon_{o}\right) /\left(\tilde{\varepsilon}_{k}+\varepsilon_{o}\right)$, $\underline{3}$ where the effective mode-dependent permittivity $\tilde{\varepsilon}_{k}$ is given by:

$$
\tilde{\varepsilon}_{k}=\varepsilon_{S}\left(1+\frac{\varepsilon_{i}-\varepsilon_{S}}{\varepsilon_{S}+\delta\left(1 / 2-\chi_{k}\right) \varepsilon_{i}+\delta\left(1 / 2+\chi_{k}\right) \varepsilon_{S}}\right)
$$

where $\delta$ is a small parameter roughly equal to the relative volume of the shell with respect to the particle. $\underline{\underline{3}}$ This equation allows a direct connection to the hybridization model $\underline{18}$

Within various ranges of frequencies, the dielectric functions have in general the form $\varepsilon=A+$ $B / W$, where $A$ and $B$ are nearly constant functions of angular frequency $\omega$, while $W$ is a fast varying function of $\omega$. In the radiofrequency domain $\varepsilon=\varepsilon-i \sigma /\left(\varepsilon_{v a c} \omega\right)$ with $\varepsilon$ the static permittivity, $\varepsilon_{v a c}$ the vacuum permittivity and $\sigma$ the DC conductivity. In the optical regime dielectrics can be successfully described by a nearly constant dielectric function $\varepsilon=\varepsilon_{d}$ and metals by a Drude dielectric function $\varepsilon=\varepsilon_{m}-\omega_{p}^{2} / \omega /(\omega+i \gamma)$, where $\varepsilon_{m}$ is the interband contribution, $\omega_{P}$ is the electron plasma frequency and $\gamma$ is the damping constant.

The frequency-dependent polarizability of a homogeneous metal nanoparticle of arbitrary shape can then be written as a sum of Drude-Lorentz terms by using expansion ??:

$$
\begin{gathered}
\alpha_{\text {plasmon }}(\omega)=\sum_{k} \frac{p_{k}\left(\varepsilon_{m}-\varepsilon_{d o}\right)}{\varepsilon_{\mathrm{eff}}}- \\
-\frac{p_{k}}{1 / 2-\chi_{k}} \frac{\varepsilon_{d o}}{\varepsilon_{\mathrm{eff}}} \frac{\widetilde{\omega}_{p k}^{2}}{\omega(\omega+i \gamma)-\tilde{\omega}_{p k}^{2}}
\end{gathered}
$$

where $\varepsilon_{d o}$ is the permittivity of the outer dielectric medium and the effective dielectric parameter is $\varepsilon_{\text {eff }}=\left(1 / 2+\chi_{k}\right) \varepsilon_{d o}+\left(1 / 2-\chi_{k}\right) \varepsilon_{m}$. For very small particles/clusters dissipation can be neglected $\left(\gamma \ll \omega_{p}\right)$ and $\tilde{\omega}_{p k}$ is the frequency of the localized plasmon resonance, which depends explicitly on the dielectric and geometric parameters of the particle: $\tilde{\omega}_{p k}^{2}=\left(1 / 2-\chi_{k}\right) \omega_{p}^{2} / \varepsilon$ eff.

Each active mode in ?? has a fast-varying (Drude-Lorentz term) factor proportional to its coupling weight $p_{k}$ and inversely proportional to $\left(1 / 2-\chi_{k}\right)$, the depolarization factor. In clustered 
particles with tight junctions, several eigenvalues get closer to $1 / 2$ and the factor $p_{k} /\left(1 / 2-\chi_{k}\right)$ has an important contribution even though the couplings $p_{k}$ are vanishingly small. The local field enhancement factor (the height of the resonance peak) is $p_{k} /\left(1 / 2-\chi_{k}\right)\left(\varepsilon_{d o} / \varepsilon_{\mathrm{eff}}\right)\left(\tilde{\omega}_{p k} / \gamma\right)$ as in the harmonic oscillator model. $\underline{\underline{22}}$ As $\chi_{k}$ approaches $1 / 2$ for tighter and tighter junctions the plasmon resonance red-shifts toward lower frequencies.

The polarizability of dielectric particles of permittivity $\varepsilon_{d i}$ covered by a thin metallic layer and embedded into a dielectric medium of permittivity $\varepsilon_{d o}$ is obtained in the first order in $\delta$, from ?? and ?? as

$$
\begin{gathered}
\alpha_{\text {shell }} \approx \sum_{k} \frac{p_{k}\left(\varepsilon_{d i}-\varepsilon_{d o}\right)}{\varepsilon_{\mathrm{eff}}}- \\
-\frac{p_{k}}{1 / 2-\chi_{k}} \frac{\varepsilon_{d o}}{\varepsilon_{\mathrm{eff}}}\left(\frac{\tilde{\omega}_{p k}^{\prime 2}}{\omega(\omega+i \gamma)-\tilde{\omega}_{p k}^{\prime 2}}+\right. \\
\left.\frac{\tilde{\omega}_{p k}^{\prime \prime 2}}{\omega(\omega+i \gamma)-\tilde{\omega}_{p k}^{\prime \prime 2}-\omega_{p}^{2} / \varepsilon_{m}}\right)
\end{gathered}
$$

with $\tilde{\omega}_{p k}^{\prime 2}=\left(\delta\left(1 / 2+\chi_{k}\right)\left(1 / 2-\chi_{k}\right) \omega_{p}^{2}\right) / \varepsilon_{\text {eff }}, \tilde{\omega}_{p k}^{\prime \prime 2}=\left(\varepsilon_{d i} / \varepsilon_{m}\right)\left(\delta\left(1 / 2-\chi_{k}\right)^{2} \omega_{p}^{2}\right) / \varepsilon_{\text {eff }}$ and $\varepsilon_{\text {eff }}=$ $\left(1 / 2+\chi_{k}\right) \varepsilon_{d o}+\left(1 / 2-\chi_{k}\right) \varepsilon_{d i}$. Like the hybridization model, $\underline{\underline{18}}$ ?? exhibits two plasmon resonances. One resonance frequency for a shelled particle is red-shifted with respect to the one of the homogenous particle by a factor of $\sqrt{\delta\left(1 / 2+\chi_{k}\right)}$. The value of the peak of the resonance is also reduced by the factor $\sqrt{\delta\left(1 / 2+\chi_{k}\right)}$. Results obtained in $\frac{19}{\underline{19}}$ can be derived from ??, by observing that $\varepsilon_{\text {eff }}$ determines the influence of $\varepsilon_{d i}$ and $\varepsilon_{d o}$ on the plasmon resonance in nanoshells. The other resonance, located at $\sqrt{\tilde{\omega}_{p k}^{\prime \prime 2}+\omega_{p}^{2} / \varepsilon_{m}}$ is blue-shifted with respect to $\tilde{\omega}_{p k}^{\prime}$ and $\omega_{p k}$ and is smaller than both the red-shifted resonance and the resonance of a similar homogeneous particle.

A straightforward application of ?? and ?? is in the design of analyte sensing. Large changes in the position of the plasmon resonance in response to small changes in the refractive index of the outer medium are desired. The refractive index $n_{d o}$ sensitivity for the wavelength $\lambda_{p k}$ of each dipole active eigenmode is $d \lambda_{p k} / d n_{d o}=\left(1 / 2+\chi_{k}\right) n_{d o} \lambda_{p k} / \varepsilon_{\text {eff }}$ which increases with increasing wavelenght and $\chi_{k}$. A body of theoretical and experimental research ${ }^{2}$ has suggested that either elongated particles or thin nanoshells have high refractive index sensitivity. A direct explanation 
of this derives directly from ?? and ?? which show the red shift of the plasmon resonance for tightly joined clusters, elongated particles and nanoshells. $\stackrel{223}{2}$ A figure of merit is given by the ratio of refractive index sensitivity over the width of the plasmon resonance. In the limit $\gamma \ll \omega_{p}$, the resonance width is given by the damping constant $\gamma$. In the case of nanoparticles $\gamma$ is given by the the damping constant of the bulk and an additional term due to the surface scattering of conduction electrons: $\gamma=v_{F}(1 / l+1 / L), 22$ with $v_{F}$ the Fermi velocity (about $1.4 \times 10^{6} \mathrm{~m} / \mathrm{s}$ for both $\mathrm{Ag}$ and $\mathrm{Au}$ ), $l$ the bulk room temperature mean free path (about $52 \mathrm{~nm}$ for $\mathrm{Ag}$ and $40 \mathrm{~nm}$ for $\mathrm{Au}$ ), and $L$ is the effective mean free path for collision with the particle's boundary. For a spherical particle of diameter $d, L=2 d / 3$, such that if $d=25 \sim 30 \mathrm{~nm}, \gamma$ is about $0.1 \mathrm{eV}$. The damping constants in nanoshells are even greater due to larger effective mean free paths for collision; $\underline{24}$ therefore nanorods are better than nanoshells when used in analyte sensing. Using ?? and ?? the refractive index sensitivity of nanoparticles can be directly calculated as a function of the aspect ratio or as a function of composition of the nanoparticle clusters.

We have analyzed clusters of nanoparticles as $n$ touching objects with a rotational surface generated by the equation: $x=g(z) \cos \phi, y=g(z) \sin \phi$ and $-L_{n} \leq z \leq L_{n}$ (the insets of Figure 1a and $1 b)$ with

$$
\begin{array}{r}
g(n, h, A, a, b, z)=A(1+\operatorname{sign}(z \operatorname{sign}(z)-(n-1) a)) \times \\
\frac{\sqrt{1-((z-\operatorname{sign}(z)(n-1) a) / a)^{2}}}{1+\left(1+b(z-\operatorname{sign}(z)(n-1) a)^{2}\right.}\left[1-\text { floor }\left(\frac{\operatorname{sign}(z) z+a}{n a}\right)\right] \times \\
{\left[h+2(A-h)\left(1-\frac{1}{1+(1-H(z))^{2}}\right)\right]}
\end{array}
$$

where $H(z)=\bmod \left((-1)^{\text {floor }(z / a)+n-1} z, a\right)^{2}, \operatorname{sign}(z)$ is signum function, $-1,0$ or 1 if $\mathrm{z}$ is negative, zero or positive, floor $(z)$ is the greatest integer less than $z$, and $\bmod (x, y)$ is the remainder of the division of $x$ by $y$. Parameters $A, a, b$ determine the shape of an individual particle, for example, the ratio $A / a$ is the aspect ratio, and $h$ determines the size of the connecting gap, and $L_{n}=n a$.

[figure][1][]1 shows the resonances in the mid-IR introduced by tight junctions because some eigenmodes have eigenvalues near $1 / 2$ and non-zero, but very small, weights. In a recent paper we 

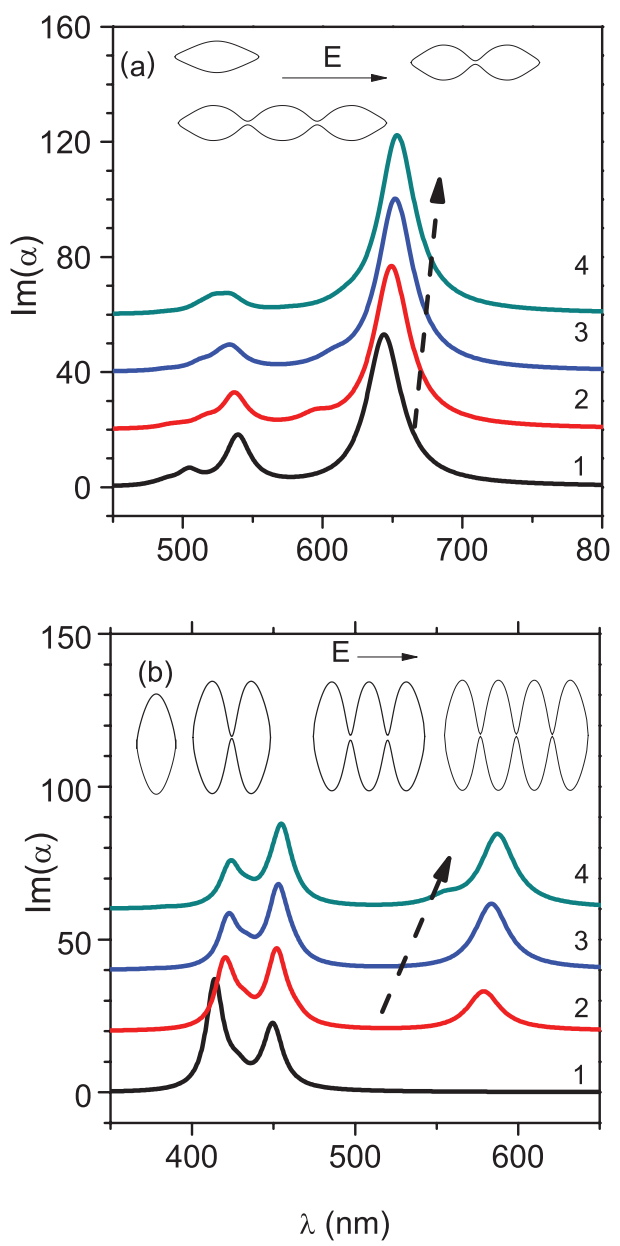

Figure 2: Imaginary part of polarizabily for clusters of elongated (a) ( $A=2, a=3, h=0.2$, $b=0.1)$ and flatten (b) $(A=3, a=2, h=0.05, b=0.1)$ particles in visible spectrum. 
have shown that in a cluster of $n$ connected particles there are $n-1$ eigenvalues close to $1 / 2$ (the smaller the junction the closer the eigenvalues). $\frac{3}{3}$ However, only the eigenmodes with antisymmetric combination of charge distribution in the lobes are active and contribute to polarizability. Thus the eigenmodes close to $1 / 2$ induce mid-IR plasmon resonances in optical spectra of metallic clusters even though the weight (dipole coupling) is quite small. In our examples clusters of two and three particles have just one resonance, while for clusters of four particles there are two overlapping resonances. As a rule, all resonances move toward infrared with increasing number of particles in the clusters, as seen in [figure][2][]2.
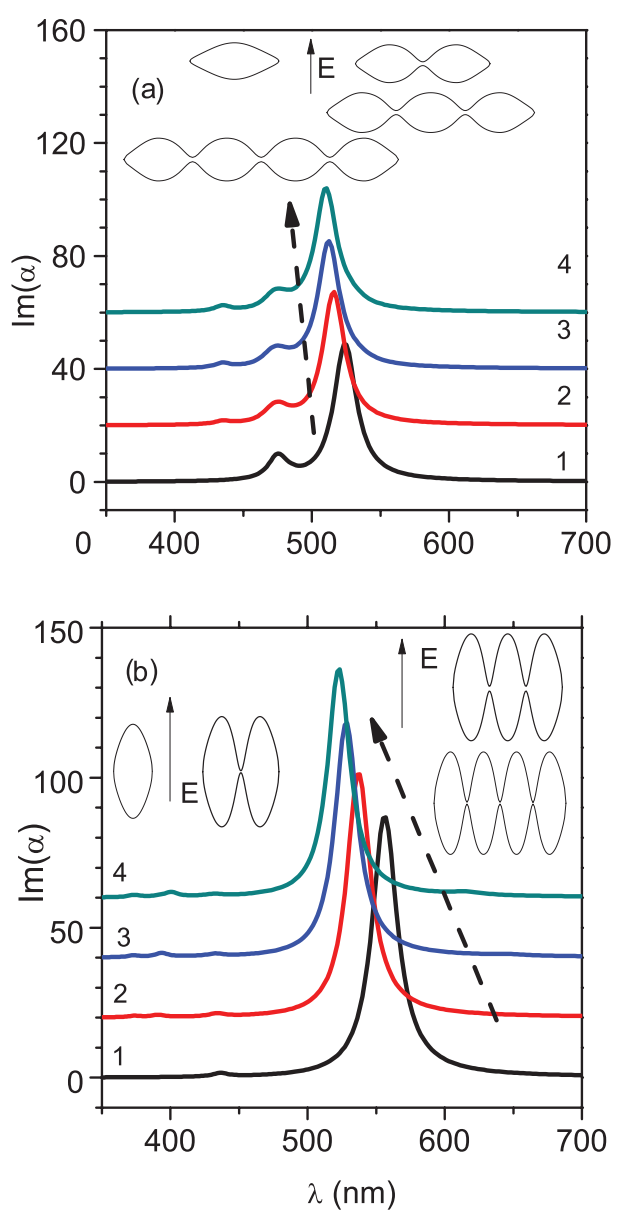

Figure 3: Imaginary part of polarizabily for clusters of elongated (a) and flatten (b) particles placed in electric perpendicular to the rotation axis.

In contrast to parallel fields $([$ figure $][1][] 1$ and $[$ figure $][2][] 2)$, the polarizability for clusters in field perpendicular to their axes, displays no new significant resonances, as seen in [figure][3][]3. 
The existing resonances move toward ultraviolet as $n$ increases. Also, the mid-IR resonance shifts toward larger wavelengths as the ratio $h / A$ decreases.

$[$ figure][2][]2 and [figure][3][]3 indicate that, in clusters as well as in individual nanoparticles, the amplitude of plasmon resonance depends also on the direction of polarization. Thus flatten particles have the greatest plasmon resonances for transverse polarization, while elongated particles has theirs for longitudinal fields. The explanation is based on the harmonic oscillator model, which relates the amplitude of plasmon resonance to the polarizability of the particles. $\underline{22}$

To conclude this letter, the spectral BIE is a rational approach which provides useful insights of the plasmonic behavior of metal nanoparticles. Linear clusters of overlapping nanoparticles are good candidates for optical sub-wavelength guides. $\stackrel{4,13}{ }$ Our results suggest that the collective resonances originating from single-particle resonances can be easily activated by exciting just one particle at one end of the cluster. In contrast, the collective resonances that are not present in an individual particle can be successfully activated by exciting the last two particles at the end of the cluster. Another possible application of linear clusters of overlapping nanoparticles is their use as optical nano-antennas instead of nanorods. ${ }^{20,21}$ The utilization of linear clusters as optical nanoantennas has a lot of promise due to recent advances in the manufacturing of highly uniform dimer arrays with gaps smaller than $10 \mathrm{~nm} .{ }^{25}$ Thus instead of using long and narrow nanorods, one can use linear clusters, with lengths comparable to the length of the nanorods and junctions comparable to the cross section of the nanorods..$^{3}$

\section{Acknowledgement}

This work has been supported by the Romanian Project "Ideas" No.120/2007 and FP 7 Nanomagma No.214107/2008.

\section{References}

(1) E. Ozbay, Science 311, 189 (2001) 
(2) P. K. Jain and M. A. El-Sayed, Chem Phys. Lett. 487, 153 (2010)

(3) T. Sandu, D. Vrinceanu and E. Gheorghiu,Phys. Rev. E 81, 021913 (2010)

(4) S. Lal, S. Link and N. J. Halas, Nat. Photonics 1 641, (2007)

(5) S. Nie and S. R. Emory, Science 275, 1102 (1997)

(6) J. Kundu, F. Le, P. Nordlander and N. J. Halas, Chem. Phys. Lett. 452, 115 (2008); F. Neubrech etal, Phys. Rev. Lett. 101, 157403 (2008); R. Adato etal, Proc. Nat. Acad. Sci. 106, 19227 (2009)

(7) J. J.Mock etal, J. Chem. Phys. 116, 6755 (2002)

(8) F. Tam, G.P. Goodrich, B.R. Johnson and N.J. Halas, Nano Lett. 7, 496 (2007)

(9) M. Danckwerts and L. Novotny, Phys. Rev. Letters 98, 026104 (2007)

(10) S. Kim, J. Jin, Y. Kim, I. Park, Y. Kim and S. Kim, Nature 453, 757 (2008)

(11) M. Quinten, Appl. Phys. B 73, 245 (2001)

(12) T. Atay, J. -H. Songa and A. V. Nurmikko, Nano Lett. 4, 1627 (2004)

(13) I. Romero, J. Aizpurua, G. W. Bryant and F. J. G. de Abajo, Optics Express 14, 9988 (2006)

(14) F. Le etal, ACS Nano 2, 707 (2008)

(15) J. B. Lassiter, J. Aizpurua, L. I. Hernandez, D. W. Brandl, I. Romero, S. Lal, J. H. Hafner, P. Nordlander and N. J. Halas, Nano Lett. 8, 1212 (2008)

(16) D. R. Fredkin and I. D. Mayergoyz, Phys. Rev. Lett. 91, 253902 (2003)

(17) I. D. Mayergoyz, D. R. Fredkin and Z. Zhang, Phys. Rev. B 72, 155412 (2005)

(18) E. Prodan, C. Radloff, N. J. Halas, P. Nordlander, Science 302, 419 (2003) 
(19) J. Zhu, Appl. Phys. Lett. 92, 241919 (2008)

(20) P. Mühlschlegel etal, Science 308, 1607 (2005)

(21) A. Alú and N. Engheta, Phys. Rev. Lett. 101, 043901 (2008)

(22) C. F. Bohren, D. R. Huffman, Absorption and Scattering of Light by Small Particles, John Wiley \& Sons, New York (1998)

(23) J. N. Anker etal, Nature Mat. 7, 442 (2008)

(24) A. Moroz, J. Phys. Chem. C 112, 10641 (2008)

(25) S. S. Aćimović etal, ACS Nano 5, 1231 (2009) 